Selcuk Journal of Agriculture and Food Sciences

http://sjafs.selcuk.edu.tr/sjafs/index

Research Article
SJAFS

(2020) 34 (1), 31-41

e-ISSN: $2458-8377$

DOI:10.15316/SJAFS.2020.192

\title{
Irrigation Water Quality Assessments for Irrigated Lands of Konya - Sarayönü Gözlü Agricultural Enterprise
}

\author{
Ahmet Melih YILMAZ ${ }^{1, *}$, Ceren Mutlu ALPÖZEN² ${ }^{2}$ Gülşah KAPLAN ${ }^{1}$ \\ ${ }^{1}$ Selçuk University, Faculty of Agriculture, Deparment of Farm Structures and Irrigation, Konya, Turkey \\ ${ }^{2} 2$. Regional Directorate of State Hydraulic Works, Operation and Maintenance Department, İzmir, Turkey
}

\begin{tabular}{l}
\hline ARTICLE INFO \\
\hline Article history: \\
Received date: 20.12 .2019 \\
Accepted date: 02.01 .2020 \\
\hline Edited by: \\
Duran YAVUZ; Selçuk University, \\
Turkey \\
Reviewed by: \\
Zeki GÖKALP; Erciyes University, \\
Turkey \\
Mehmet ŞAHIN; Selçuk University, \\
Turkey \\
\hline Keywords: \\
Irrigation \\
Irrigation Water Quality \\
Soil Salinity \\
Salinity \\
\hline
\end{tabular}

\section{Introduction}

Soil and water resources are the most important sources of wealth of the countries. Today, majority of these sources are under the threat of extinction, thus identification of available potentials and preservation of such potential are quite a significant issue. Water is an essential component of human life. Together with developing technologies, unconscious uses and rapid pollution, ever-increasing populations and rapid depletion of fresh and clean water resources have brought the water into the first place in world agenda. Agriculture is the greatest water user sector (about $70 \%$ of freshwater resources are used in agriculture) and it is respectively followed by industrial and domestic uses. Efficient use and recycle are the primary ways to be followed for sustainability of water resources. Irrigation; is defined as the artificial supply of partial quantity of water needed by the plants, but not fully met by natural precipitations to root zones of the plants in a controlled fashion (Kara 2005).

\footnotetext{
* Corresponding author email: afyilmaz@selcuk.edu.tr

* This study is a part of master thesis of Ceren Mutlu Alpözen.
}

Water and soil quality (salinity) are the primary issues to be considered for sustainability of agriculture. Ongoing climate changes and increasing water use exert serious threats on water resources. Groundwater resources are more influenced by such threats and under qualitative and quantitative degradation. Such a degradation is more prominent in "Konya Closed Basin" without any replenishment from outside and with large irrigated fields. Various salts re transferred to soils through irrigation water. Then, salinity problems emerge based on quality parameters of water resources. Poor-quality water may terminate agricultural production if the relevant measures were not taken (Taş et al 2013).

Type and quantity of dissolved substances in water influence irrigation water quality.With the analyses conducted on irrigation waters, total concentrations of salts and quantity of different elements are identified. Drought and salinity stress are the primary limiting factors in front of agricultural production. Especially the salinity levels of greater than $15 \mathrm{dS} / \mathrm{m}$ may result in serious yields losses (Husain et al 2003).

Total salt concentration of irrigation waters is expressed as electrical conductivity (EC x 106) ( $\mu \mathrm{mhos} / \mathrm{cm} ; 1000 \mu \mathrm{mhos} / \mathrm{cm}=1 \mathrm{mmhos} / \mathrm{cm}=1 \mathrm{dS} / \mathrm{m}$ ). 
Irrigation waters successfully used in irrigated farming usually have a total salt concentration lower than 2250 $\mu \mathrm{mhos} / \mathrm{cm}$. However, water with an electrical conductivity of less than $750 \mu \mathrm{mhos} / \mathrm{cm}$ are recommended to be used in irrigated farming. The waters with electrical conductivity values of between $750-2250 \mu \mathrm{mhos} / \mathrm{cm}$ are also largely used without a significant lose in yield under proper drainage and operational conditions, but in case of insufficient or poor drainage conditions, such waters may result in serious salinity problems in irrigated lands (Ayyıldız 1983).

Anliatamer (2007), conducted a study to investigate soil salinity in irrigation district of Ankara Haymana Türkşerefli Earth-fill Dam and reported increased salinity levels in Babayakup Stream jointing with a tributary of Şerefli Creek within the research site. Researcher indicated that measures should be taken while using this water in irrigated farming and high salinity levels in some sections of the research site were mainly attributed to unconscious irrigation practices and excessive water application rates in surface irrigations of the farmers.

Yeter \& Yurtseven (2015) conducted a study to investigate the effects of different quality irrigation waters on alfalfa and reported a recess in plant growth, significant decreases in yield and quality with saline water irrigations. On the other hand, plant growth and development returned to normal levels when the leaching was performed. It was concluded that for high yield levels in alfalfa, irrigation water salinity should be less than $1.5 \mathrm{dSm}^{-1}$.

Salts generate osmatic pressure in soil solution and influence plant water use accordingly. Plant water use decreases under high osmotic pressure and such a case then ends up with plant die up. Therefore, total salt concentration of irrigation waters is generally used as classification criterion for irrigation water quality (Yurtseven 2016).

Gürcan (2016) conducted a study in irrigation district of irrigation cooperative of Soğulca village of Haymana, Ankara about quality of water resources and classified irrigation waters as $\mathrm{C}_{3}$ (highly saline) and indicated that this quality irrigation water should not be used in poorly-drained sections. It was also indicated that salinity problem was not observed in irrigated lands of the study area despite the salinity problems of irrigation waters, then recommended the construction of closed or open drainage facilities to prevent potential salinity problems in the future.

Minareci \& Öztürk (2012), investigated boron concentrations of water samples taken from the reservoirs of Sevişler Dam, Demirköprü Dam, Avşar Dam and Gölmarmara Dam in Manisa province and reported boron concentrations as between $0.008-3.066 \mathrm{mg} / \mathrm{L}$.

Korkmaz et al (2016) conducted a study in Right Bank Irrigation district of Menemen and indicated that improper irrigation methods and low irrigatino water application efficiency raised groundwater levels. High groundwater tables negatively influence plant cultiva- tion, so they recommended the construction of proper drainage facilitiesor improvement of already existing facilities.

Dorak \& Çelik (2017) conducted a study to determine the effects of domestic and industrial wastewaters on water quality of Nilüfer Stream. Researchers took water samples from effluent discharge points of 5 wastewater treatment plants in 4 periods between $\mathrm{Au}-$ gust 2013 and May 2014. Wastewater quality parameters varied based on sampling periods, watersamples were classified as between $\mathrm{C}_{2} \mathrm{~S}_{1}-\mathrm{C}_{4} \mathrm{~S}_{4}$ based on EC and $\mathrm{SAR}$ values. Water samples taken before and after the discharge points revealed that treatment plant effluents negatively influenced $\mathrm{pH}, \mathrm{EC}$, ammonia, phosphorus, sulphate, boron and chlorine values of Nilüfer Stream.

\section{Materials and Methods}

Water samples were taken from 20 deep wells selected from irrigated fields of Konya - Sarayönü Gözlü Agricultural Enterprise in intensive irrigation periods (May - September) and soil samples were taken from irrigated fields.

Konya province is located between $36^{\circ} 41^{1}$ and $39^{\circ}$ $16^{1}$ north latitudes and between $31^{\circ} 14^{1}$ and $34^{\circ} 26^{1}$ east longitudes. Average altitude is $1.016 \mathrm{~m}$. Konya is surrounded by Ankara and Eskişehir from the north, Isparta and Afyonkarahisar from the west, Antalya, Karaman and Mersin from the south and Niğde and Aksaray provinces from the east. Total surface area of the province is $41.001 \mathrm{~km}^{2}$ (Anonymous 2016).

Gözlü Agricultural Enterprise is located $78 \mathrm{~km}$ from Konya and $28 \mathrm{~km}$ from Sarayönü town. The enterprise is surrounded by Özkent district from the east, Gözlü district from the West, Kolukısa district from the South, Çeşmelisebil District from the north and Başkuyu district from the northwest. There is a stabilized rood connection of $34 \mathrm{~km}$ to Altınova Agricultural Enterprise and there is about 7-8 km distance from the fields of Konuklar Agricultural Enterprise. Location of the research site is presented in Figure 1.

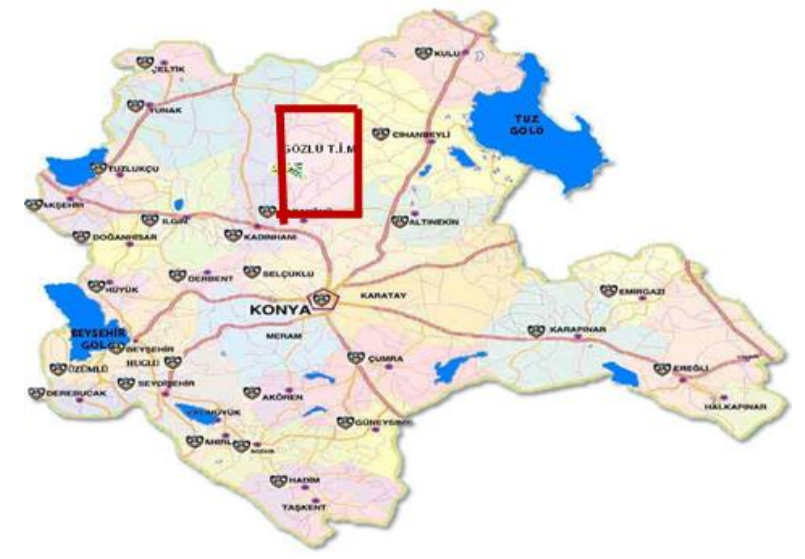

Figure 1

Location of study area

The research site has a terrestrial climate with cold and precipitated winters and hot and dry summers. 
Precipitations are mostly observed in winter and spring months. Wind speed may reach to $100-120 \mathrm{~km} / \mathrm{h}$ in March and April. Such high wind speeds accelerate wind erosion process. Therefore, $225 \mathrm{~km}$ forest line was constructed for erosion prevention.

Konya province with a surface area of $41.001 \mathrm{~km}^{2}$ has the largest fields of the country. Cereals (wheat, barley, oat, rye) are cultivated over the majority of these lands. Edible legumes (dry bean, lentils, chickpea), oil crops (sunflower, opium), industrial crops (sugar beet, potato) and feed crops (alfalfa, vetch, silage maize) are also cultivated in the region (Anonymous 2013).

Apart from these crops, fruits (pear, apple, plum, apricot, cherry, peach, melon, watermelon, sour cherry, walnut, strawberry, grape) and vegetables (tomato, cucumber, pepper, fresh bean, eggplant, cabbage, lettuce, spinach, carrot) are also cultivated in the region.

Gözlü Agricultural Enterprise has 288.303,5 da lands and irrigated farming is practiced over 35.129 da of these lands. Wheat, barley, alfalfa, vetch, maize and sunflower are cultivated over the agricultural fields of Gözlü Agricultural Enterprise.

There are 93 deep wells over 35.129 da irrigated fields of Gözlü Agricultural Enterprise and irrigation water is supplied from these wells. Wells were started to be opened in 2006 and continued until 2013.

Irrigations are performed with sprinkler irrigation, drip irrigation and self-propelled irrigation systems. There aren't any drainage canals around the irrigated fields of the enterprise. Sprinkler irrigation is used over the majority of irrigated lands and the rest is irrigated with drip irrigation, linear-move and center-pivot selfpropelled irrigation systems.

Water samples were taken from 20 deep wells selected among 93 wells opened by the enterprise and actively operating through purposeful sampling procedure during the intensive irrigation season (May September). Water samples were taken in accordance with the principles specified in Sağlam (1978).

Samples were brought to laboratory, filtered, placed into clean glass bottles and preserved in a fridge until the time of analysis. Well depths varied between $160-$ $260 \mathrm{~m}$ and well discharges varied between $20-53.2$ $1 / \mathrm{s}$.

Soil samples were taken from the fields irrigated from the selected wells in a season with the most intensive irrigation and the greatest capillary salt transport (July). Disturbed and undisturbed soil samples were taken from 6 different points with a bucket auger at 3 different depth segments $(0-30,30-60$ and 60-90 cm). Samples were brought to laboratory, air dried and passed through $2 \mathrm{~mm}$ sieve, placed into nylon bags and preserved in a fridge until the time of analysis. Water and soil sampling locations are presented in Figure 2.

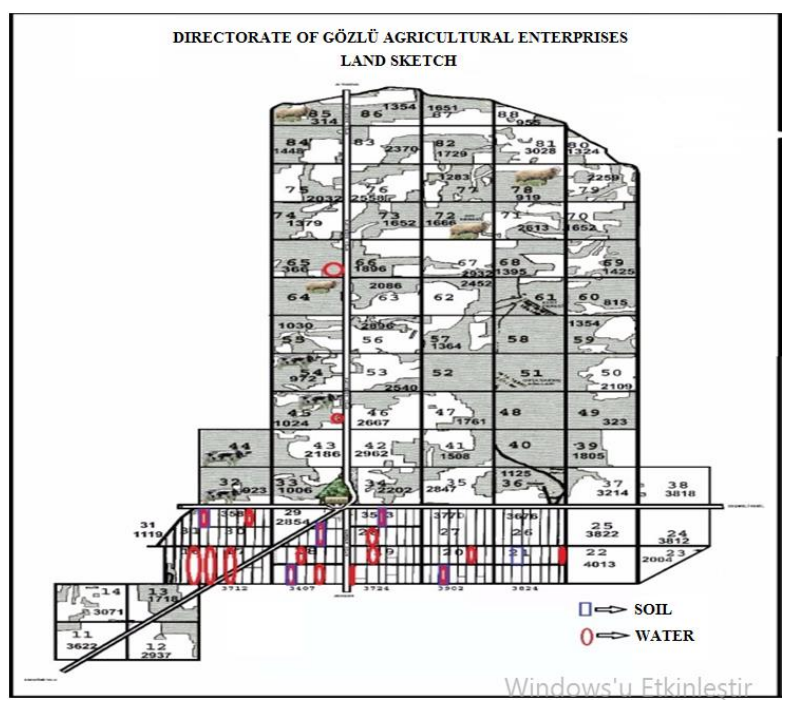

Figure 2

Water and soil sampling locations

\section{Results and Discussion}

Water samples were taken throughout the irrigation season in May, June, July, August and September. Irrigation water chemical analysis results for May are provided in Table 1. Irrigation water $\mathrm{pH}$ values varied between 6.80 - 7.37, Ec values varied between 1158 $1490 \mu \mathrm{mhos} / \mathrm{cm}$. Considering the water-soluble anion and cations, $\mathrm{Ca}^{+2}$ was the dominant cation and $\mathrm{SO}_{4}^{-2}$ was the dominant anion. There were no residual sodium carbonate (RSC) in irrigation waters. Sodium adsorption ratios (SAR) varied between $1.04-1.40$, \% $\mathrm{Na}$ values varied between 18.1 - 24.5 and boron concentrations varied between $0.15-0.23 \mathrm{ppm}$. Water samples taken in May was classified as $\mathrm{C}_{3} \mathrm{~S}_{1}$ according to US Salinity Lab classification system.EC values of irrigation water samples based on well numbers are presented in Figure 3. The greatest salinity values were observed in 10, 14 and 4-numbered wells and all water samples had an EC value of greater than allowable limit value (750 $\mu \mathrm{mhos} / \mathrm{cm})$.

Boron concentrations of water samples are presented in Figure 4. All samples had a boron concentration of lower than allowable limit value $(0.7 \mathrm{ppm})$ and there were not any problems with regard to boron concentrations in May. 
Table 1

Irrigation water chemical analysis results for May

\begin{tabular}{|c|c|c|c|c|c|c|c|c|c|c|c|c|c|c|c|c|c|}
\hline \multirow{3}{*}{$\begin{array}{c}\text { Well } \\
\text { No }\end{array}$} & \multirow{3}{*}{ pH } & \multirow{3}{*}{$\begin{array}{c}\mathrm{EC} \times 10^{6} \\
\mu \mathrm{mhos} / \mathrm{cm} \\
25^{\circ} \mathrm{C}\end{array}$} & \multicolumn{10}{|c|}{ WATER SOLUBLE } & \multirow[b]{3}{*}{ RSC } & \multirow[b]{3}{*}{ SAR } & \multirow[b]{3}{*}{$\% \mathrm{Na}$} & \multirow{3}{*}{$\begin{array}{c}\text { Irrigation } \\
\text { water } \\
\text { class }\end{array}$} & \multirow{3}{*}{$\begin{array}{l}\text { Boron } \\
(\mathbf{p p m})\end{array}$} \\
\hline & & & \multicolumn{5}{|c|}{ Cations (me/l) } & \multicolumn{5}{|c|}{ Anions (me/l) } & & & & & \\
\hline & & & $\mathrm{Na}^{+}$ & $\mathbf{K}^{+}$ & $\mathrm{Ca}^{+2}$ & $\mathrm{Mg}^{+2}$ & Total & $\mathrm{CO}_{2}{ }^{-}$ & $\mathrm{HCO}_{3}^{-}$ & $\mathrm{Cl}^{-}$ & $\mathrm{SO}_{2}{ }^{-}$ & Total & & & & & \\
\hline 1 & 6,80 & 1220 & 2,69 & 0,19 & 6,59 & 3,18 & 12,65 & - & 4,34 & 1,50 & 5,89 & 11,73 & - & 1,21 & 21,2 & $\mathrm{C}_{3} \mathrm{~S}_{1}$ & 0,15 \\
\hline 2 & 7,10 & 1405 & 2,80 & 3,23 & 5,59 & 3,31 & 14,93 & - & 4,76 & 1,65 & 6,75 & 13,16 & - & 1,32 & 18,7 & $\mathrm{C}_{3} \mathrm{~S}_{1}$ & 0,20 \\
\hline 3 & 6,85 & 1428 & 2,90 & 4,30 & 5,21 & 3,31 & 15,72 & - & 4,42 & 1,55 & 6,79 & 12,76 & - & 1,40 & 18,4 & $\mathrm{C}_{3} \mathrm{~S}_{1}$ & 0,20 \\
\hline 4 & 7,37 & 1470 & 3,18 & 0,21 & 7,35 & 3,47 & 14,21 & - & 5,62 & 2,55 & 6,91 & 15,08 & - & 1,36 & 22,3 & $\mathrm{C}_{3} \mathrm{~S}_{1}$ & 0,21 \\
\hline 5 & 7,01 & 1231 & 2,65 & 0,19 & 5,84 & 3,42 & 12,10 & - & 4,30 & 1,70 & 6,51 & 12,51 & - & 1,23 & 21,9 & $\mathrm{C}_{3} \mathrm{~S}_{1}$ & 0,20 \\
\hline 6 & 7,03 & 1230 & 2,79 & 0,19 & 5,05 & 3,36 & 11,39 & - & 4,33 & 1,60 & 6,94 & 12,87 & - & 1,36 & 24,4 & $\mathrm{C}_{3} \mathrm{~S}_{1}$ & 0,19 \\
\hline 7 & 6,89 & 1402 & 2,76 & 0,20 & 6,97 & 3,47 & 13,40 & - & 6,05 & 1,45 & 6,85 & 14,35 & - & 1,20 & 20,5 & $\mathrm{C}_{3} \mathrm{~S}_{1}$ & 0,20 \\
\hline 8 & 6,95 & 1415 & 2,75 & 0,19 & 7,83 & 3,33 & 14,10 & - & 5,32 & 1,45 & 7,35 & 14,12 & - & 1,16 & 19,5 & $\mathrm{C}_{3} \mathrm{~S}_{1}$ & 0,19 \\
\hline 9 & 7,30 & 1445 & 2,89 & 0,20 & 7,32 & 3,38 & 13,79 & - & 5,72 & 1,55 & 7,36 & 14,62 & - & 1,24 & 20,9 & $\mathrm{C}_{3} \mathrm{~S}_{1}$ & 0,21 \\
\hline 10 & 7,03 & 1490 & 2,87 & 0,21 & 8,05 & 3,43 & 14,56 & - & 6,29 & 1,60 & 7,11 & 15,00 & - & 1,19 & 19,7 & $\mathrm{C}_{3} \mathrm{~S}_{1}$ & 0,22 \\
\hline 11 & 7,05 & 1410 & 2,86 & 0,21 & 7,48 & 3,46 & 14,01 & - & 4,75 & 1,30 & 8,32 & 14,37 & - & 1,22 & 20,4 & $\mathrm{C}_{3} \mathrm{~S}_{1}$ & 0,22 \\
\hline 12 & 6,96 & 1278 & 2,86 & 0,20 & 5,96 & 3,41 & 12,43 & - & 4,46 & 1,20 & 7,47 & 13,13 & - & 1,32 & 23,0 & $\mathrm{C}_{3} \mathrm{~S}_{1}$ & 0,22 \\
\hline 13 & 6,91 & 1355 & 2,87 & 0,21 & 6,76 & 3,46 & 13,30 & - & 4,20 & 1,60 & 7,99 & 13,79 & - & 1,26 & 21,5 & $\mathrm{C}_{3} \mathrm{~S}_{1}$ & 0,23 \\
\hline 14 & 6,88 & 1485 & 2,89 & 0,21 & 8,15 & 3,40 & 14,65 & - & 5,72 & 1,55 & 7,78 & 15,05 & - & 1,20 & 19,7 & $\mathrm{C}_{3} \mathrm{~S}_{1}$ & 0,22 \\
\hline 15 & 7,13 & 1392 & 2,41 & 0,20 & 7,25 & 3,40 & 13,26 & - & 5,01 & 1,45 & 7,70 & 14,16 & - & 1,04 & 18,1 & $\mathrm{C}_{3} \mathrm{~S}_{1}$ & 0,22 \\
\hline 16 & 7,01 & 1255 & 2,93 & 0,21 & 5,42 & 3,45 & 12,01 & - & 4,54 & 1,00 & 7,36 & 12,90 & - & 1,39 & 24,3 & $\mathrm{C}_{3} \mathrm{~S}_{1}$ & 0,23 \\
\hline 17 & 7,13 & 1374 & 2,90 & 0,20 & 6,92 & 3,41 & 13,43 & - & 5,15 & 1,30 & 7,40 & 13,85 & - & 1,27 & 21,5 & $\mathrm{C}_{3} \mathrm{~S}_{1}$ & 0,22 \\
\hline 18 & 7,18 & 1260 & 2,87 & 0,24 & 5,94 & 3,49 & 12,54 & - & 4,35 & 1,20 & 7,19 & 12,74 & - & 1,32 & 22,8 & $\mathrm{C}_{3} \mathrm{~S}_{1}$ & 0,23 \\
\hline 19 & 7,11 & 1232 & 2,85 & 0,20 & 5,72 & 3,41 & 12,18 & - & 3,60 & 1,40 & 7,51 & 12,51 & - & 1,33 & 23,3 & $\mathrm{C}_{3} \mathrm{~S}_{1}$ & 0,21 \\
\hline 20 & 7,35 & 1158 & 2,72 & 0,19 & 4,83 & 3,36 & 11,10 & - & 3,80 & 1,35 & 7,03 & 12,18 & - & 1,34 & 24,5 & $\mathrm{C}_{3} \mathrm{~S}_{1}$ & 0,20 \\
\hline
\end{tabular}

Irrigation water chemical analysis results for June are provided in Table 2. Irrigation water $\mathrm{pH}$ values varied between $6.87-7.45$, EC values varied between $1071-1711 \mu \mathrm{mhos} / \mathrm{cm}$. Considering the water-soluble anion and cations, $\mathrm{Ca}^{+2}$ was the dominant cation and $\mathrm{SO}_{4}^{-2}$ was the dominant anion. There were no residual sodium carbonate (RSC) in irrigation waters. Sodium adsorption ratios (SAR) varied between $1.05-1.34$, \% $\mathrm{Na}$ values varied between $16.9-25.0$ and boron concentrations varied between $0.15-0.23 \mathrm{ppm}$. Water samples taken in May was classified as $\mathrm{C}_{3} \mathrm{~S}_{1}$ according to US Salinity Lab classification system.

The greatest salinity values were observed in 4,17 and 16-numbered wells and all water samples had an EC value of greater than allowable limit value (750 $\mu \mathrm{mhos} / \mathrm{cm})$.

All samples had a boron concentration of lower than allowable limit value $(0.7 \mathrm{ppm})$ and there were not any problems with regard to boron concentrations in June.

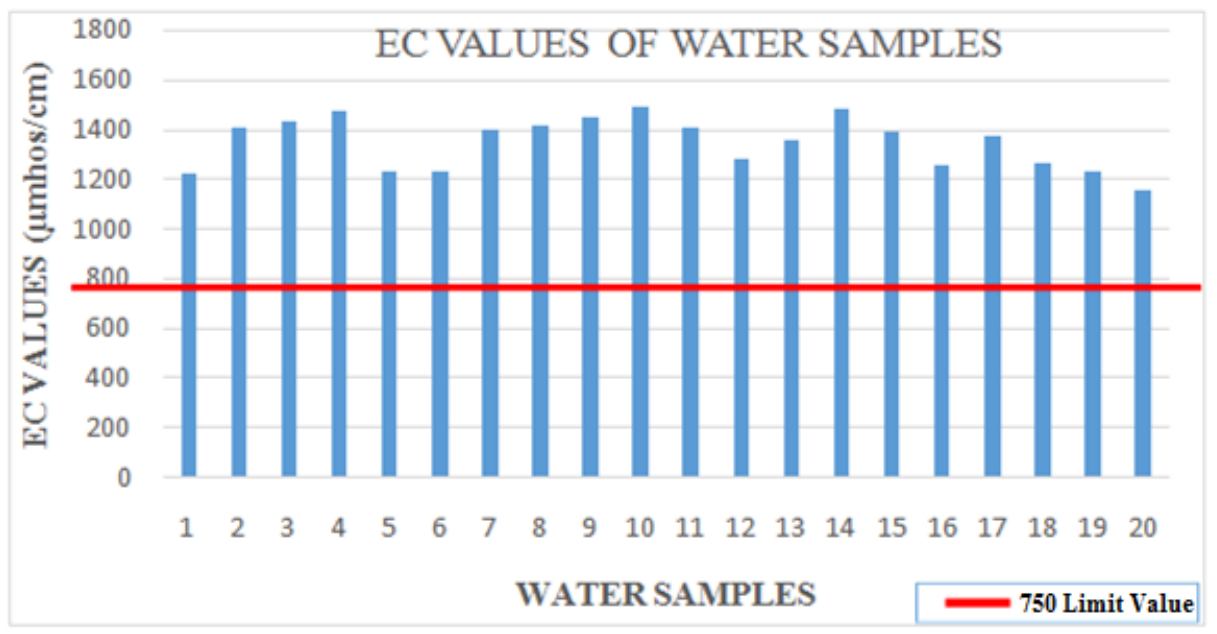

Figure 3

EC values of water samples in May 


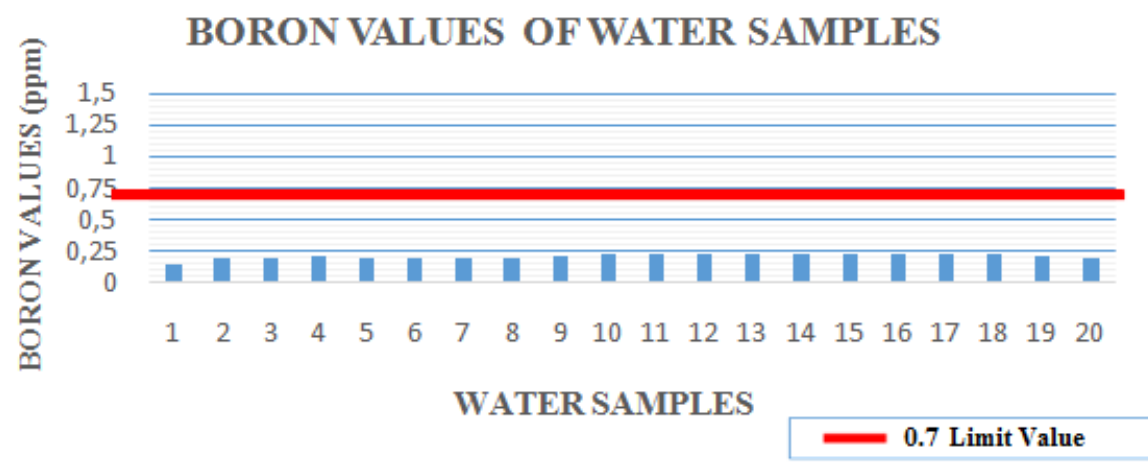

Figure 4

Boron concentrations of water samples in May

Irrigation water chemical analysis results for July are provided in Table 3. Irrigation water $\mathrm{pH}$ values varied between $6.86-7.39$, EC values varied between $1440-1771 \mu \mathrm{mhos} / \mathrm{cm}$. Considering the water-soluble anion and cations, $\mathrm{Ca}^{+2}$ was the dominant cation and $\mathrm{HCO}_{3}{ }^{-}$and $\mathrm{SO}_{4}{ }^{-2}$ were the dominant anion. There were no residual sodium carbonate (RSC) in irrigation waters. Sodium adsorption ratios (SAR) varied between $1.17-1.36, \% \mathrm{Na}$ values varied between $17.7-21.9$ and boron concentrations varied between $0.28-0.35$ ppm. Water samples taken in May was classified as

Table 2

Irrigation water chemical analysis results for June
$\mathrm{C}_{3} \mathrm{~S}_{1}$ according to US Salinity Lab classification system.

EC values of irrigation water samples based on well numbers are presented in Figure 5. The greatest salinity values were observed in 9, 12, 11-numbered wells and all water samples had an EC value of greater than allowable limit value $(750 \mu \mathrm{mhos} / \mathrm{cm})$.

Boron concentrations of water samples are presented in Figure 6. All samples had a boron concentration of lower than allowable limit value $(0.7 \mathrm{ppm})$ and there were not any problems with regard to boron concentrations in May.

\begin{tabular}{|c|c|c|c|c|c|c|c|c|c|c|c|c|c|c|c|c|c|}
\hline \multirow[b]{2}{*}{ WellNo } & \multirow[b]{2}{*}{$\mathbf{p H}$} & \multirow{2}{*}{ 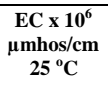 } & \multicolumn{10}{|c|}{ WATER SOLUBLE } & \multirow[b]{2}{*}{ RSC } & \multirow[b]{2}{*}{ SAR } & \multirow[b]{2}{*}{$\% \mathrm{Na}$} & \multirow{2}{*}{$\begin{array}{l}\text { Irrigation } \\
\text { Water } \\
\text { Class }\end{array}$} & \multirow{2}{*}{$\begin{array}{l}\text { Boron } \\
(\mathrm{ppm})\end{array}$} \\
\hline & & & $\mathrm{Na}^{+}$ & $\mathbf{K}^{+}$ & $\frac{C_{a t i o n s}}{\mathbf{C a}^{+2}}$ & $\mathbf{M g}^{+2}$ & $\Sigma$ & $\mathrm{CO}_{3}^{-2}$ & $\frac{\mathrm{A}}{\mathrm{HCO}_{3}{ }^{-}}$ & $\frac{\mathrm{ons}(\mathrm{m}}{\mathrm{Cl}}$ & $\mathrm{SO}_{4}^{-2}$ & $\Sigma$ & & & & & \\
\hline 1 & 7,34 & 1117 & 2,62 & 0,17 & 4,96 & 3,24 & 10,99 & - & 4,00 & 1,85 & 5,38 & 11,23 & - & 1,29 & 23,8 & $\mathrm{C}_{3} \mathrm{~S}_{1}$ & 0,19 \\
\hline 2 & 7,25 & 1460 & 2,83 & 0,24 & 8,06 & 3,33 & 14,46 & - & 6,14 & 1,80 & 6,94 & 14,88 & - & 1,18 & 19,5 & $\mathrm{C}_{3} \mathrm{~S}_{1}$ & 0,21 \\
\hline 3 & 7,19 & 1259 & 2,80 & 0,20 & 5,98 & 3,34 & 12,32 & - & 4,75 & 1,90 & 6,15 & 12,80 & - & 1,29 & 22,7 & $\mathrm{C}_{3} \mathrm{~S}_{1}$ & 0,20 \\
\hline 4 & 6,88 & 1711 & 3,34 & 0,23 & 10,08 & 3,49 & 17,14 & - & 7,77 & 2,35 & 7,08 & 17,20 & - & 1,28 & 19,4 & $\mathrm{C}_{3} \mathrm{~S}_{1}$ & 0,23 \\
\hline 5 & 7,02 & 1539 & 2,73 & 0,18 & 9,80 & 3,28 & 15,99 & - & 7,70 & 1,60 & 6,59 & 15,89 & - & 1,06 & 17,0 & $\mathrm{C}_{3} \mathrm{~S}_{1}$ & 0,19 \\
\hline 6 & 6,89 & 1404 & 2,80 & 0,20 & 6,81 & 3,33 & 13,14 & - & 5,60 & 1,70 & 6,77 & 14,07 & - & 1,24 & 21,3 & $\mathrm{C}_{3} \mathrm{~S}_{1}$ & 0,19 \\
\hline 7 & 7,03 & 1189 & 2,71 & 0,20 & 5,35 & 3,46 & 11,72 & - & 5,11 & 1,65 & 5,15 & 11,91 & - & 1,29 & 23,1 & $\mathrm{C}_{3} \mathrm{~S}_{1}$ & 0,20 \\
\hline 8 & 6,93 & 1547 & 2,81 & 0,19 & 9,01 & 3,31 & 15,32 & - & 6,99 & 1,85 & 7,13 & 15,97 & - & 1,13 & 18,3 & $\mathrm{C}_{3} \mathrm{~S}_{1}$ & 0,19 \\
\hline 9 & 7,14 & 1296 & 2,80 & 0,20 & 5,37 & 3,31 & 11,68 & - & 4,65 & 1,70 & 5,97 & 12,32 & - & 1,34 & 23,9 & $\mathrm{C}_{3} \mathrm{~S}_{1}$ & 0,19 \\
\hline 10 & 6,97 & 1184 & 2,73 & 0,20 & 5,28 & 3,36 & 11,57 & - & 4,65 & 1,40 & 6,60 & 12,65 & - & 1,31 & 22,6 & $\mathrm{C}_{3} \mathrm{~S}_{1}$ & 0,19 \\
\hline 11 & 6,90 & 1210 & 2,75 & 0,19 & 5,58 & 3,40 & 11,92 & - & 4,20 & 1,90 & 6,42 & 12,52 & - & 1,29 & 23,0 & $\mathrm{C}_{3} \mathrm{~S}_{1}$ & 0,20 \\
\hline 12 & 6,91 & 1398 & 2,79 & 0,20 & 6,85 & 3,35 & 13,19 & - & 5,44 & 1,60 & 7,07 & 14,11 & - & 1,23 & 21,1 & $\mathrm{C}_{3} \mathrm{~S}_{1}$ & 0,20 \\
\hline 13 & 6,88 & 1225 & 2,75 & 0,20 & 5,76 & 3,32 & 12,03 & - & 4,54 & 1,55 & 6,72 & 12,81 & - & 1,29 & 22,8 & $\mathrm{C}_{3} \mathrm{~S}_{1}$ & 0,19 \\
\hline 14 & 6,87 & 1425 & 2,71 & 0,20 & 7,36 & 3,29 & 13,56 & - & 5,45 & 1,90 & 7,06 & 14,41 & - & 1,17 & 19,9 & $\mathrm{C}_{3} \mathrm{~S}_{1}$ & 0,18 \\
\hline 15 & 6,99 & 1313 & 2,61 & 0,19 & 7,23 & 3,25 & 13,28 & - & 5,63 & 1,45 & 6,83 & 13,91 & - & 1,14 & 19,6 & $\mathrm{C}_{3} \mathrm{~S}_{1}$ & 0,17 \\
\hline 16 & 6,94 & 1612 & 2,92 & 0,21 & 10,15 & 3,39 & 16,67 & - & 8,00 & 1,55 & 7,20 & 16,75 & - & 1,12 & 17,5 & $\mathrm{C}_{3} \mathrm{~S}_{1}$ & 0,21 \\
\hline 17 & 7,01 & 1614 & 2,71 & 0,19 & 9,81 & 3,28 & 15,99 & - & 8,16 & 1,80 & 6,73 & 16,69 & - & 1,05 & 16,9 & $\mathrm{C}_{3} \mathrm{~S}_{1}$ & 0,17 \\
\hline 18 & 7,06 & 1071 & 2,59 & 0,20 & 4,20 & 3,33 & 10,32 & - & 4,41 & 1,95 & 5,17 & 11,53 & - & 1,33 & 25,0 & $\mathrm{C}_{3} \mathrm{~S}_{1}$ & 0,17 \\
\hline 19 & 7,14 & 1406 & 2,55 & 0,17 & 8,10 & 3,20 & 14,02 & - & 6,60 & 1,90 & 6,49 & 14,99 & - & 1,07 & 18,1 & $\mathrm{C}_{3} \mathrm{~S}_{1}$ & 0,15 \\
\hline 20 & 7,45 & 1266 & 2,49 & 0,18 & 6,97 & 3,19 & 12,83 & - & 5,86 & 1,85 & 5,32 & 13,03 & - & 1,10 & 19,4 & $\mathrm{C}_{3} \mathrm{~S}_{1}$ & 0,15 \\
\hline
\end{tabular}


Yılmaz et al. / Selcuk J Agr Food Sci, (2020) 34 (1), 31-41

Table 3

Irrigation water chemical analysis results for July

\begin{tabular}{|c|c|c|c|c|c|c|c|c|c|c|c|c|c|c|c|c|c|}
\hline \multirow{3}{*}{$\begin{array}{l}\text { Well } \\
\text { No }\end{array}$} & \multirow{3}{*}{ pH } & \multirow{3}{*}{$\begin{array}{c}\mathrm{EC} \times 10^{6} \\
\mu \mathrm{mhos} / \mathrm{cm} \\
25^{\circ} \mathrm{C}\end{array}$} & \multicolumn{10}{|c|}{ WATER SOLUBLE } & \multirow{3}{*}{ RSC } & \multirow{3}{*}{ SAR } & \multirow{3}{*}{$\% \mathrm{Na}$} & \multirow{3}{*}{$\begin{array}{c}\text { Irrigation } \\
\text { Water } \\
\text { Class }\end{array}$} & \multirow{3}{*}{$\begin{array}{l}\text { Boron } \\
(\mathbf{p p m})\end{array}$} \\
\hline & & & \multicolumn{5}{|c|}{ Cations (me/l) } & \multicolumn{5}{|c|}{ Anions (me/l) } & & & & & \\
\hline & & & $\mathrm{Na}^{+}$ & $\mathbf{K}^{+}$ & $\mathrm{Ca}^{+2}$ & $\mathbf{M g}^{+2}$ & $\Sigma$ & $\mathrm{CO}_{3}^{-2}$ & $\mathrm{HCO}_{3}^{-}$ & $\mathrm{Cl}^{-}$ & $\mathrm{SO}_{4}^{-2}$ & $\Sigma$ & & & & & \\
\hline 1 & 7,22 & 1440 & 2,91 & 0,12 & 8,75 & 3,18 & 14,96 & - & 6,02 & 2,05 & 5,98 & 14,05 & - & 1,19 & 19,4 & $\mathrm{C}_{3} \mathrm{~S}_{1}$ & 0,28 \\
\hline 2 & 6,99 & 1598 & 3,05 & 0,14 & 9,69 & 3,28 & 16,16 & - & 7,71 & 1,60 & 6,66 & 15,97 & - & 1,19 & 18,8 & $\mathrm{C}_{3} \mathrm{~S}_{1}$ & 0,32 \\
\hline 3 & 7,39 & 1553 & 3,02 & 0,13 & 9,34 & 3,27 & 15,76 & - & 6,90 & 2,45 & 6,53 & 15,88 & - & 1,20 & 19,1 & $\mathrm{C}_{3} \mathrm{~S}_{1}$ & 0,31 \\
\hline 4 & 7,27 & 1574 & 3,47 & 0,17 & 9,49 & 3,38 & 16,51 & - & 6,62 & 2,50 & 6,78 & 15,90 & - & 1,36 & 21,0 & $\mathrm{C}_{3} \mathrm{~S}_{1}$ & 0,35 \\
\hline 5 & 6,97 & 1585 & 3,00 & 0,13 & 9,89 & 3,25 & 16,27 & - & 7,06 & 1,70 & 6,98 & 15,74 & - & 1,17 & 18,4 & $\mathrm{C}_{3} \mathrm{~S}_{1}$ & 0,31 \\
\hline 6 & 6,88 & 1603 & 3,18 & 0,15 & 10,18 & 3,32 & 16,83 & - & 7,81 & 2,10 & 6,72 & 16,63 & - & 1,22 & 18,8 & $\mathrm{C}_{3} \mathrm{~S}_{1}$ & 0,32 \\
\hline 7 & 7,00 & 1596 & 3,19 & 0,17 & 8,95 & 3,45 & 15,76 & - & 7,31 & 2,25 & 6,74 & 16,30 & - & 1,28 & 20,2 & $\mathrm{C}_{3} \mathrm{~S}_{1}$ & 0,35 \\
\hline 8 & 6,93 & 1644 & 3,22 & 0,16 & 10,62 & 3,32 & 17,32 & - & 7,39 & 2,25 & 7,33 & 16,97 & - & 1,21 & 18,5 & $\mathrm{C}_{3} \mathrm{~S}_{1}$ & 0,33 \\
\hline 9 & 6,98 & 1771 & 3,22 & 0,17 & 11,10 & 3,31 & 17,80 & - & 8,58 & 2,55 & 6,93 & 18,06 & - & 1,19 & 18,0 & $\mathrm{C}_{3} \mathrm{~S}_{1}$ & 0,33 \\
\hline 10 & 6,97 & 1622 & 3,20 & 0,17 & 9,69 & 3,40 & 16,46 & - & 7,69 & 1,90 & 6,78 & 16,37 & - & 1,25 & 19,4 & $\mathrm{C}_{3} \mathrm{~S}_{1}$ & 0,34 \\
\hline 11 & 6,91 & 1701 & 3,16 & 0,16 & 11,06 & 3,38 & 17,76 & - & 7,50 & 1,85 & 7,82 & 17,17 & - & 1,17 & 17,7 & $\mathrm{C}_{3} \mathrm{~S}_{1}$ & 0,32 \\
\hline 12 & 6,89 & 1710 & 3,16 & 0,16 & 10,64 & 3,32 & 17,28 & - & 7,26 & 1,65 & 7,90 & 16,81 & - & 1,19 & 18,2 & $\mathrm{C}_{3} \mathrm{~S}_{1}$ & 0,32 \\
\hline 13 & 6,86 & 1697 & 3,22 & 0,17 & 10,30 & 3,36 & 17,05 & - & 6,98 & 1,55 & 8,35 & 16,88 & - & 1,23 & 18,8 & $\mathrm{C}_{3} \mathrm{~S}_{1}$ & 0,33 \\
\hline 14 & 6,90 & 1630 & 3,16 & 0,16 & 9,91 & 3,31 & 16,54 & - & 6,80 & 2,35 & 6,98 & 16,13 & - & 1,22 & 19,1 & $\mathrm{C}_{3} \mathrm{~S}_{1}$ & 0,31 \\
\hline 15 & 7,10 & 1654 & 3,21 & 0,16 & 10,33 & 3,32 & 17,02 & - & 6,92 & 1,80 & 7,74 & 16,46 & - & 1,22 & 18,8 & $\mathrm{C}_{3} \mathrm{~S}_{1}$ & 0,31 \\
\hline 16 & 6,95 & 1615 & 3,26 & 0,18 & 9,72 & 3,37 & 16,53 & - & 6,86 & 1,50 & 7,40 & 15,76 & - & 1,27 & 19,7 & $\mathrm{C}_{3} \mathrm{~S}_{1}$ & 0,32 \\
\hline 17 & 7,04 & 1513 & 3,23 & 0,18 & 8,49 & 3,40 & 15,30 & - & 6,30 & 2,10 & 6,45 & 14,85 & - & 1,32 & 21,1 & $\mathrm{C}_{3} \mathrm{~S}_{1}$ & 0,32 \\
\hline 18 & 7,26 & 1503 & 3,24 & 0,18 & 7,82 & 3,51 & 14,75 & - & 6,90 & 2,05 & 6,32 & 15,27 & - & 1,36 & 21,9 & $\mathrm{C}_{3} \mathrm{~S}_{1}$ & 0,33 \\
\hline 19 & 7,06 & 1616 & 3,15 & 0,15 & 10,73 & 3,31 & 17,34 & - & 6,10 & 1,75 & 8,48 & 16,33 & - & 1,18 & 18,1 & $\mathrm{C}_{3} \mathrm{~S}_{1}$ & 0,30 \\
\hline 20 & 7,29 & 1573 & 3,08 & 0,14 & 9,68 & 3,31 & 16,21 & - & 7,30 & 1,55 & 6,65 & 15,50 & - & 1,20 & 19,0 & $\mathrm{C}_{3} \mathrm{~S}_{1}$ & 0,30 \\
\hline
\end{tabular}

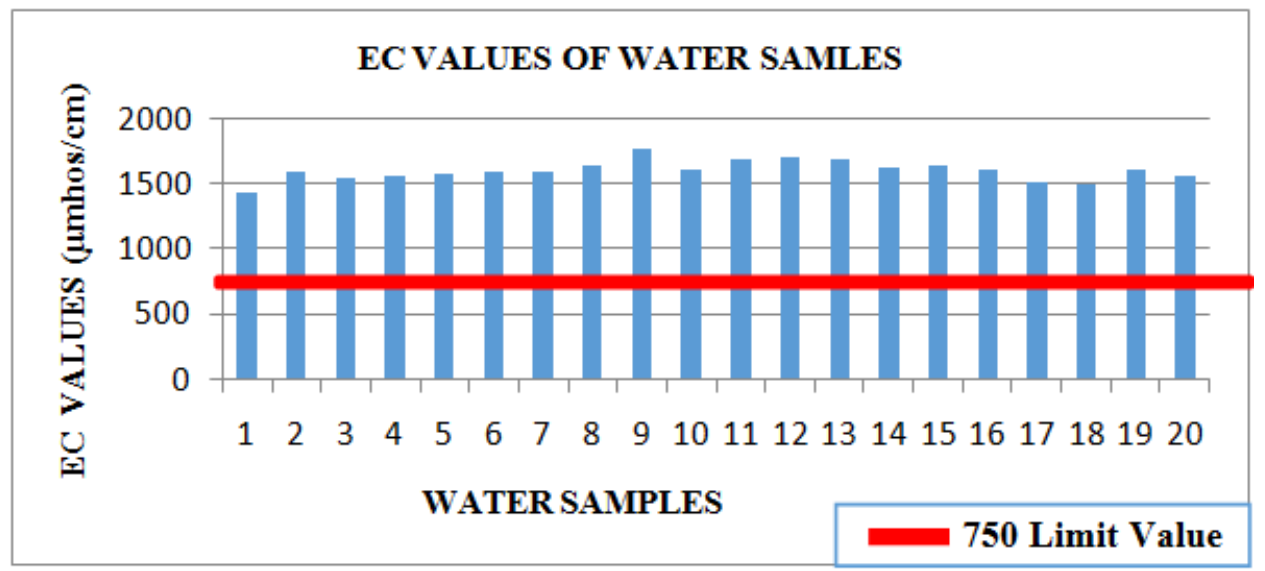

Figure 5

EC values of water samples in July 
Y1lmaz et al. / Selcuk J Agr Food Sci, (2020) 34 (1), 31-41

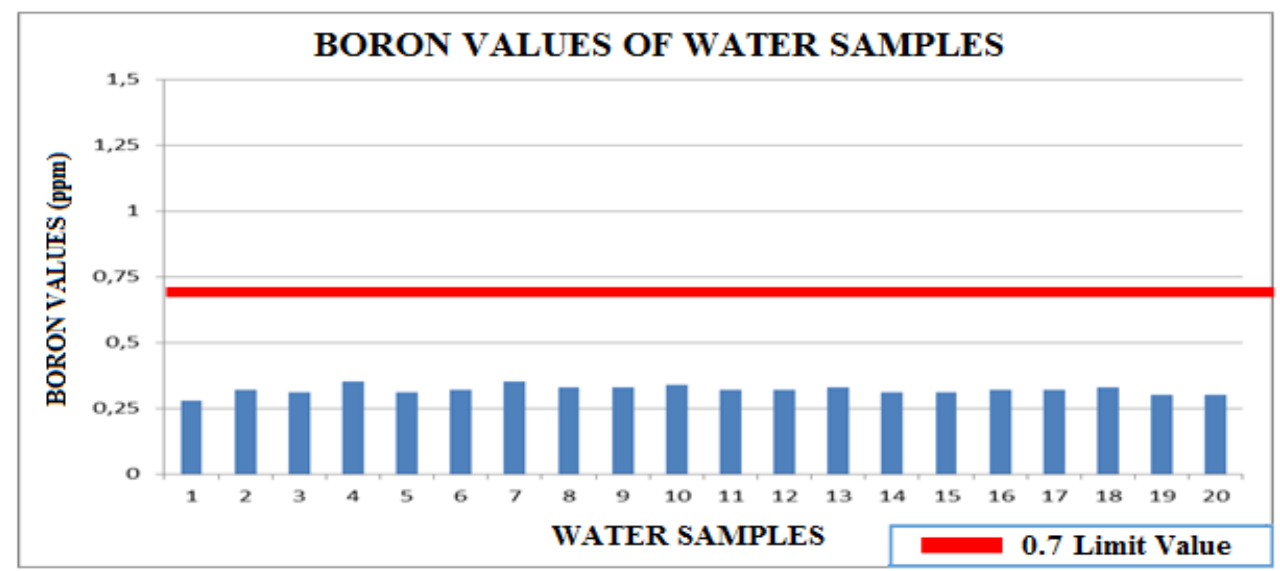

Figure 6

Boron concentrations of water samples in July

Irrigation water chemical analysis results for $\mathrm{Au}-$ gust are provided in Table 4. Irrigation water $\mathrm{pH}$ values varied between $6.08-7.30$, EC values varied between 1692 - $1989 \mu \mathrm{mhos} / \mathrm{cm}$. Considering the water-soluble anion and cations, $\mathrm{Ca}^{+2}$ was the dominant cation and $\mathrm{HCO}_{3}{ }^{-}$was the dominant anion. There were no residual sodium carbonate (RSC) in irrigation waters. Sodium adsorption ratios (SAR) varied between $1.00-1.16$, \% $\mathrm{Na}$ values varied between $15.1-17.8$ and boron concentrations varied between $0.27-0.36 \mathrm{ppm}$. Water samples taken in May was classified as $\mathrm{C}_{3} \mathrm{~S}_{1}$ according to US Salinity Lab classification system.

The greatest salinity values were observed in 11, 14 and 4-numbered wells and all water samples had an EC value of greater than allowable limit value (750 $\mu \mathrm{mhos} / \mathrm{cm})$.

All samples had a boron concentration of lower than allowable limit value $(0.7 \mathrm{ppm})$ and there were not any problems with regard to boron concentrations in August.

Table 4

Irrigation water chemical analysis results for August
Irrigation water chemical analysis results for September are provided in Table 5. Irrigation water $\mathrm{pH}$ values varied between $6.84-7.26$, EC values varied between $1474-1946 \mu \mathrm{mhos} / \mathrm{cm}$. Considering the water-soluble anion and cations, $\mathrm{Ca}^{+2}$ was the dominant cation and $\mathrm{HCO}_{3}{ }^{-}$was the dominant anion. There were no residual sodium carbonate (RSC) in irrigation waters. Sodium adsorption ratios (SAR) varied between $1.04-1.21, \% \mathrm{Na}$ values varied between $15.1-19.7$ and boron concentrations varied between $0.21-0.33$ ppm. Water samples taken in May was classified as $\mathrm{C}_{3} \mathrm{~S}_{1}$ according to US Salinity Lab classification system.EC values of irrigation water samples based on well numbers are presented in Figure 7 . The greatest salinity values were observed in 8,11 and 15numbered wells and all water samples had an EC value of greater than allowable limit value $(750 \mu \mathrm{mhos} / \mathrm{cm})$.

Boron concentrations of water samples are presented in Figure 8. All samples had a boron concentration of lower than allowable limit value $(0.7 \mathrm{ppm})$ and there were not any problems with regard to boron concentrations in August.

\begin{tabular}{|c|c|c|c|c|c|c|c|c|c|c|c|c|c|c|c|c|c|}
\hline \multirow{3}{*}{$\begin{array}{l}\text { Well } \\
\text { No }\end{array}$} & \multirow{3}{*}{ pH } & \multirow{3}{*}{$\begin{array}{c}\mathrm{EC} \times 10^{6} \\
\mu \mathrm{mhos} / \mathrm{cm} \\
25^{\circ} \mathrm{C}\end{array}$} & \multicolumn{10}{|c|}{ WATER SOLUBLE } & \multirow{3}{*}{ RSC } & \multirow{3}{*}{ SAR } & \multirow{3}{*}{$\% \mathrm{Na}$} & \multirow{3}{*}{$\begin{array}{c}\text { Irrigation } \\
\text { Water } \\
\text { Class }\end{array}$} & \multirow{3}{*}{$\begin{array}{l}\text { Boron } \\
(\mathbf{p p m})\end{array}$} \\
\hline & & & \multicolumn{5}{|c|}{ Cations (me/l) } & \multicolumn{5}{|c|}{ Anions (me/l) } & & & & & \\
\hline & & & $\mathrm{Na}^{+}$ & $\mathbf{K}^{+}$ & $\mathbf{C a}^{+2}$ & $\mathbf{M g}^{+2}$ & $\Sigma$ & $\mathrm{CO}_{3}{ }^{-2}$ & $\mathrm{HCO}_{3}{ }^{-}$ & $\mathrm{Cl}^{-}$ & $\mathrm{SO}_{4}^{-2}$ & $\Sigma$ & & & & & \\
\hline 1 & 7,30 & 1741 & 2,85 & 0,11 & 9,73 & 4,45 & 17,14 & - & 8,31 & 3,00 & 6,48 & 17,79 & - & 1,07 & 16,6 & $\mathrm{C}_{3} \mathrm{~S}_{1}$ & 0,27 \\
\hline 2 & 6,97 & 1865 & 3,02 & 0,14 & 10,99 & 4,61 & 18,76 & - & 9,37 & 2,40 & 6,92 & 18,69 & - & 1,08 & 16,0 & $\mathrm{C}_{3} \mathrm{~S}_{1}$ & 0,31 \\
\hline 3 & 7,06 & 1767 & 2,92 & 0,13 & 10,79 & 4,63 & 18,47 & - & 8,54 & 1,20 & 7,95 & 17,69 & - & 1,05 & 15,8 & $\mathrm{C}_{3} \mathrm{~S}_{1}$ & 0,29 \\
\hline 4 & 7,03 & 1924 & 3,10 & 0,14 & 11,06 & 4,71 & 19,01 & - & 9,78 & 1,50 & 7,13 & 18,41 & - & 1,10 & 16,3 & $\mathrm{C}_{3} \mathrm{~S}_{1}$ & 0,31 \\
\hline 5 & 7,10 & 1725 & 2,71 & 0,15 & 8,50 & 5,09 & 16,45 & - & 9,35 & 2,00 & 6,22 & 17,57 & - & 1,03 & 16,4 & $\mathrm{C}_{3} \mathrm{~S}_{1}$ & 0,34 \\
\hline 6 & 6,82 & 1809 & 3,03 & 0,13 & 10,69 & 4,68 & 18,53 & - & 9,19 & 2,30 & 6,59 & 18,08 & - & 1,09 & 16,3 & $\mathrm{C}_{3} \mathrm{~S}_{1}$ & 0,31 \\
\hline 7 & 6,92 & 1821 & 2,97 & 0,14 & 10,33 & 4,86 & 18,30 & - & 9,91 & 2,00 & 6,73 & 18,64 & - & 1,07 & 16,2 & $\mathrm{C}_{3} \mathrm{~S}_{1}$ & 0,33 \\
\hline 8 & 6,82 & 1909 & 2,95 & 0,13 & 11,55 & 4,68 & 19,31 & - & 9,26 & 3,00 & 6,93 & 19,19 & - & 1,03 & 15,2 & $\mathrm{C}_{3} \mathrm{~S}_{1}$ & 0,31 \\
\hline 9 & 6,92 & 1827 & 3,02 & 0,14 & 10,96 & 4,65 & 18,77 & - & 8,72 & 1,50 & 8,32 & 18,54 & - & 1,08 & 16,0 & $\mathrm{C}_{3} \mathrm{~S}_{1}$ & 0,32 \\
\hline 10 & 6,86 & 1784 & 2,93 & 0,14 & 10,44 & 4,81 & 18,32 & - & 8,41 & 2,30 & 6,87 & 17,58 & - & 1,06 & 15,9 & $\mathrm{C}_{3} \mathrm{~S}_{1}$ & 0,36 \\
\hline 11 & 6,89 & 1989 & 3,03 & 0,14 & 12,09 & 4,79 & 20,05 & - & 9,53 & 2,60 & 7,21 & 19,34 & - & 1,04 & 15,1 & $\mathrm{C}_{3} \mathrm{~S}_{1}$ & 0,30 \\
\hline 12 & 6,98 & 1906 & 3,04 & 0,14 & 11,04 & 4,68 & 18,90 & - & 8,75 & 3,80 & 6,52 & 19,07 & - & 1,08 & 16,0 & $\mathrm{C}_{3} \mathrm{~S}_{1}$ & 0,32 \\
\hline 13 & 6,75 & 1871 & 3,01 & 0,14 & 11,19 & 4,70 & 19,04 & - & 8,04 & 2,50 & 7,85 & 18,39 & - & 1,06 & 15,8 & $\mathrm{C}_{3} \mathrm{~S}_{1}$ & 0,32 \\
\hline 14 & 6,80 & 1937 & 3,00 & 0,14 & 11,50 & 4,71 & 19,35 & - & 8,94 & 3,50 & 7,22 & 19,66 & - & 1,05 & 15,5 & $\mathrm{C}_{3} \mathrm{~S}_{1}$ & 0,32 \\
\hline 15 & 6,98 & 1860 & 2,97 & 0,14 & 11,36 & 4,59 & 19,06 & - & 9,52 & 2,90 & 5,81 & 18,23 & - & 1,05 & 15,5 & $\mathrm{C}_{3} \mathrm{~S}_{1}$ & 0,29 \\
\hline 16 & 6,99 & 1810 & 3,14 & 0,17 & 9,80 & 4,85 & 17,96 & - & 8,80 & 2,30 & 7,20 & 18,30 & - & 1,16 & 17,4 & $\mathrm{C}_{3} \mathrm{~S}_{1}$ & 0,27 \\
\hline 17 & 7,01 & 1692 & 3,03 & 0,15 & 9,09 & 4,74 & 17,01 & - & 8,21 & 2,00 & 6,65 & 16,86 & - & 1,15 & 17,8 & $\mathrm{C}_{3} \mathrm{~S}_{1}$ & 0,32 \\
\hline 18 & 6,08 & 1807 & 3,05 & 0,15 & 9,09 & 4,86 & 17,15 & - & 8,90 & 2,60 & 7,28 & 18,78 & - & 1,15 & 17,7 & $\mathrm{C}_{3} \mathrm{~S}_{1}$ & 0,31 \\
\hline 19 & 7,01 & 1823 & 2,81 & 0,12 & 10,90 & 4,63 & 18,46 & - & 8,58 & 1,70 & 7,49 & 17,77 & - & 1,00 & 15,2 & $\mathrm{C}_{3} \mathrm{~S}_{1}$ & 0,29 \\
\hline 20 & 6,78 & 1879 & 2,97 & 0,14 & 11,08 & 4,64 & 18,83 & - & 8,81 & 2,60 & 7,16 & 18,57 & - & 1,05 & 15,7 & $\mathrm{C}_{3} \mathrm{~S}_{1}$ & 0,31 \\
\hline
\end{tabular}


Table 5

Irrigation water chemical analysis results for September

\begin{tabular}{|c|c|c|c|c|c|c|c|c|c|c|c|c|c|c|c|c|c|}
\hline \multirow[b]{2}{*}{$\begin{array}{c}\text { Well } \\
\text { No }\end{array}$} & \multirow[b]{2}{*}{ pH } & \multirow{2}{*}{$\begin{array}{c}\mathrm{EC} \mathrm{x} \mathrm{10} 0^{6} \\
\mu \mathrm{mhos} / \mathrm{cm} \\
25^{\circ} \mathrm{C} \\
\end{array}$} & \multicolumn{10}{|c|}{ WATER SOLUBLE } & \multirow[b]{2}{*}{ RSC } & \multirow[b]{2}{*}{ SAR } & \multirow[b]{2}{*}{$\% \mathrm{Na}$} & \multirow{2}{*}{$\begin{array}{c}\text { Irrigation } \\
\text { Water } \\
\text { Class }\end{array}$} & \multirow[b]{2}{*}{$\begin{array}{l}\text { Boron } \\
(\mathrm{ppm})\end{array}$} \\
\hline & & & $\mathrm{Na}^{+}$ & $\mathbf{K}^{+}$ & $\begin{array}{l}\text { tions }(\mathrm{m} \\
\mathrm{Ca}^{+2}\end{array}$ & $\mathrm{Mg}^{+2}$ & Total & $\mathrm{CO}_{3}^{-2}$ & $\begin{array}{r}\mathrm{A} \\
\mathrm{HCO}_{3}{ }^{-} \\
\end{array}$ & $\frac{\mathrm{ons}(\mathrm{m}}{\mathrm{Cl}^{-}}$ & 1) $\mathrm{SO}_{4}{ }^{-2}$ & Total & & & & & \\
\hline 1 & 7,26 & 1549 & 2,75 & 0,10 & 8,69 & 4,40 & 15,94 & - & 7,02 & 1,70 & 6,67 & 15,39 & - & 1,07 & 17,2 & $\mathrm{C}_{3} \mathrm{~S}_{1}$ & 0,21 \\
\hline 2 & 7,17 & 1775 & 2,98 & 0,13 & 10,79 & 4,63 & 18,53 & - & 7,98 & 2,90 & 6,77 & 17,65 & - & 1,07 & 16,0 & $\mathrm{C}_{3} \mathrm{~S}_{1}$ & 0,27 \\
\hline 3 & 7,24 & 1864 & 2,90 & 0,12 & 10,50 & 4,59 & 18,11 & - & 9,61 & 3,20 & 6,02 & 18,83 & - & 1,05 & 16,0 & $\mathrm{C}_{3} \mathrm{~S}_{1}$ & 0,26 \\
\hline 4 & 7,04 & 1894 & 3,11 & 0,14 & 11,06 & 4,70 & 19,01 & - & 8,46 & 2,20 & 8,08 & 18,74 & - & 1,10 & 16,3 & $\mathrm{C}_{3} \mathrm{~S}_{1}$ & 0,29 \\
\hline 5 & 7,08 & 1672 & 2,74 & 0,15 & 8,33 & 5,09 & 16,31 & - & 8,03 & 1,40 & 7,65 & 17,08 & - & 1,05 & 16,7 & $\mathrm{C}_{3} \mathrm{~S}_{1}$ & 0,32 \\
\hline 6 & 6,87 & 1795 & 3,05 & 0,14 & 10,56 & 4,68 & 18,43 & - & 8,36 & 2,30 & 6,89 & 17,55 & - & 1,10 & 16,5 & $\mathrm{C}_{3} \mathrm{~S}_{1}$ & 0,28 \\
\hline 7 & 7,24 & 1474 & 2,95 & 0,16 & 6,58 & 5,26 & 14,95 & - & 7,60 & 1,20 & 6,22 & 15,02 & - & 1,21 & 19,7 & $\mathrm{C}_{3} \mathrm{~S}_{1}$ & 0,33 \\
\hline 8 & 6,93 & 1946 & 3,08 & 0,14 & 11,53 & 4,67 & 19,42 & - & 8,83 & 2,70 & 8,53 & 20,06 & - & 1,08 & 15,8 & $\mathrm{C}_{3} \mathrm{~S}_{1}$ & 0,28 \\
\hline 9 & 6,95 & 1854 & 3,03 & 0,14 & 11,41 & 4,74 & 19,32 & - & 8,48 & 3,20 & 6,85 & 18,53 & - & 1,06 & 15,6 & $\mathrm{C}_{3} \mathrm{~S}_{1}$ & 0,29 \\
\hline 10 & 6,97 & 1895 & 3,03 & 0,15 & 10,26 & 4,79 & 18,23 & - & 9,09 & 3,30 & 6,68 & 19,07 & - & 1,10 & 16,6 & $\mathrm{C}_{3} \mathrm{~S}_{1}$ & 0,30 \\
\hline 11 & 6,84 & 1921 & 3,03 & 0,14 & 12,04 & 4,82 & 20,03 & - & 8,88 & 2,70 & 8,25 & 19,83 & - & 1,04 & 15,1 & $\mathrm{C}_{3} \mathrm{~S}_{1}$ & 0,31 \\
\hline 12 & 7,00 & 1789 & 3,04 & 0,14 & 10,82 & 4,68 & 18,68 & - & 8,20 & 1,20 & 7,99 & 17,39 & - & 1,09 & 16,2 & $\mathrm{C}_{3} \mathrm{~S}_{1}$ & 0,30 \\
\hline 13 & 6,92 & 1840 & 3,05 & 0,15 & 10,83 & 4,76 & 18,79 & - & 8,73 & 3,40 & 6,92 & 19,05 & - & 1,09 & 16,2 & $\mathrm{C}_{3} \mathrm{~S}_{1}$ & 0,29 \\
\hline 14 & 6,92 & 1833 & 3,00 & 0,14 & 11,08 & 4,67 & 18,89 & - & 8,53 & 2,80 & 7,76 & 19,09 & - & 1,06 & 15,8 & $\mathrm{C}_{3} \mathrm{~S}_{1}$ & 0,28 \\
\hline 15 & 6,96 & 1896 & 3,10 & 0,14 & 11,85 & 4,69 & 19,78 & - & 8,17 & 2,40 & 7,98 & 18,55 & - & 1,07 & 15,6 & $\mathrm{C}_{3} \mathrm{~S}_{1}$ & 0,30 \\
\hline 16 & 6,97 & 1774 & 3,13 & 0,16 & 9,53 & 4,88 & 17,70 & - & 9,25 & 2,00 & 7,50 & 18,75 & - & 1,16 & 17,6 & $\mathrm{C}_{3} \mathrm{~S}_{1}$ & 0,31 \\
\hline 17 & 6,92 & 1688 & 3,05 & 0,16 & 9,09 & 4,78 & 17,08 & - & 7,54 & 1,20 & 7,82 & 16,56 & - & 1,15 & 17,8 & $\mathrm{C}_{3} \mathrm{~S}_{1}$ & 0,31 \\
\hline 18 & 7,04 & 1776 & 3,02 & 0,16 & 9,85 & 4,86 & 17,89 & - & 9,36 & 2,50 & 6,63 & 18,49 & - & 1,11 & 16,8 & $\mathrm{C}_{3} \mathrm{~S}_{1}$ & 0,31 \\
\hline 19 & 7,08 & 1805 & 2,97 & 0,13 & 11,01 & 4,63 & 18,74 & - & 8,34 & 1,90 & 7,60 & 17,84 & - & 1,06 & 15,8 & $\mathrm{C}_{3} \mathrm{~S}_{1}$ & 0,28 \\
\hline 20 & 6,87 & 1869 & 2,96 & 0,13 & 10,91 & 4,63 & 18,63 & - & 8,36 & 2,70 & 7,72 & 18,78 & - & 1,06 & 15,8 & $\mathrm{C}_{3} \mathrm{~S}_{1}$ & 0,28 \\
\hline
\end{tabular}

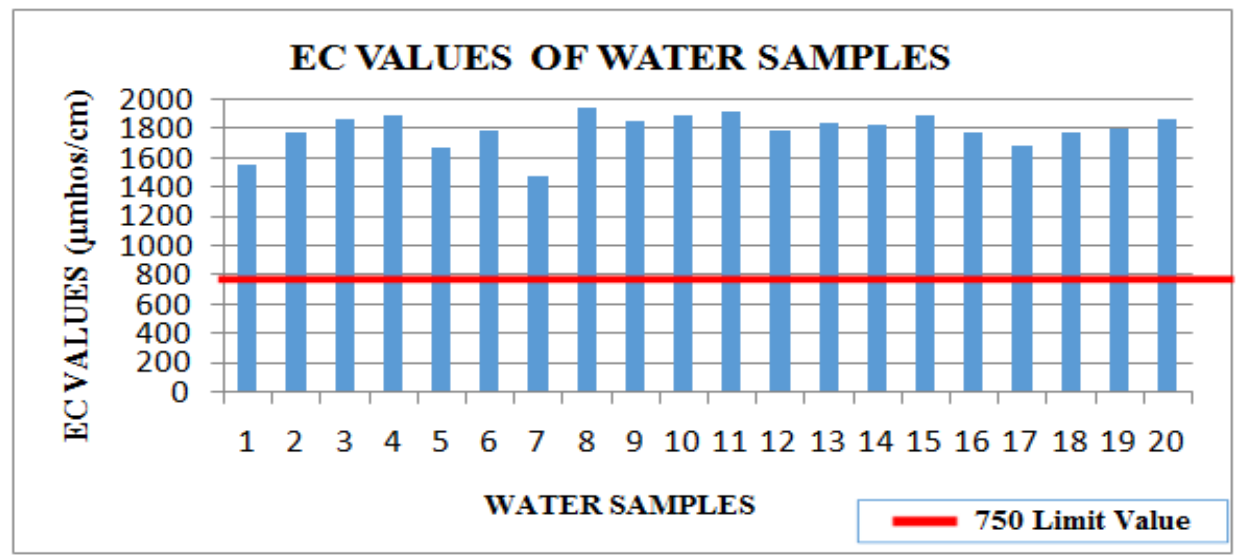

Figure 7

EC values of water samples in September

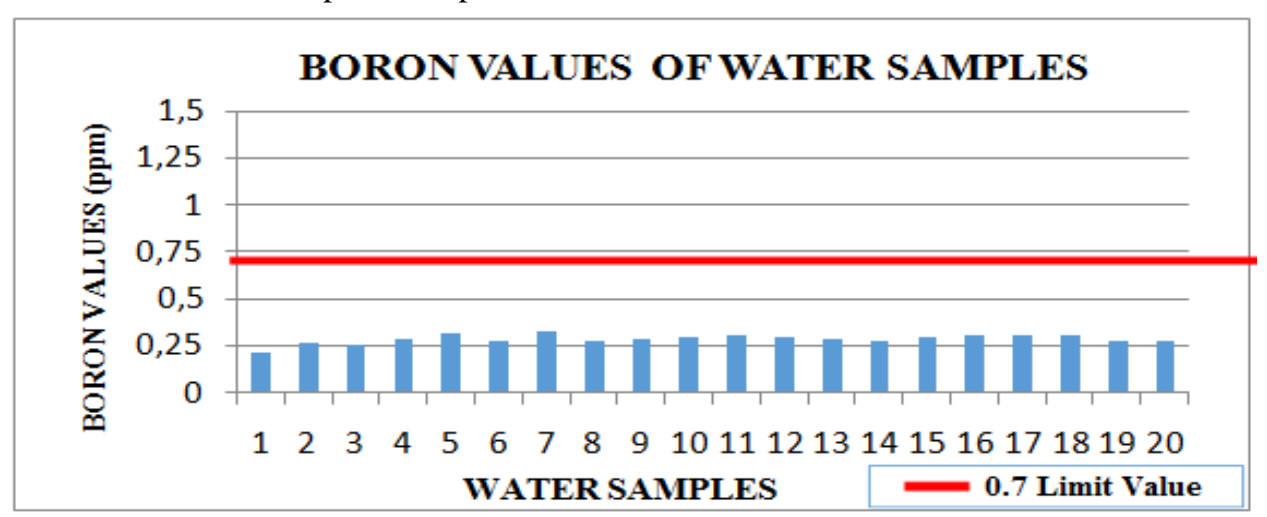

Figure 8

Boron concentrations of water samples in September

Results of physical analyses conducted on disturbed and undisturbed soil samples taken from $0-30 \mathrm{~cm}, 30-$ $60 \mathrm{~cm}$ and $60-90 \mathrm{~cm}$ depths are provided in Table 6.Degree of saturation values varied between $46.2-$ $57.4 \%$, field capacity (FC) values varied between 26.1
- 38.7\%, permanent wilting point (PWP) values varied between $14.2-21.3 \%$ and unit weights varied between $1.36-1.49 \mathrm{~g} / \mathrm{cm}^{3}$, soil texture was clay $(\mathrm{C})$ and clayloam (CL). 
Results of chemical analyses conducted on soil samples are provided in Table 7. Soil $\mathrm{pH}$ values varied between $7.11-7.90$, salinity values varied between $696-803 \mu \mathrm{mhos} / \mathrm{cm}$. Salinity values all layers were below the soil salinity threshold value (4000 $\mu \mathrm{mhos} / \mathrm{cm})$. With regard to water soluble cations and anions, $\mathrm{Na}^{+}$and $\mathrm{Ca}^{+2}$ were the dominant cations and $\mathrm{SO}_{4}{ }^{-2}$ was the dominant anion. Cation Exchange capacity (CEC) of soil samples varied between 15.19-17.23 me/100g. Exchangeable sodium percentages (ESP) varied between $6.60-7.49 \%$ and all values were below the threshold ESP value (15\%). Lime contents varied between $1.58-12.94 \%$ and boron concentrations varied between $0.08-0.85 \mathrm{ppm}$ and all values were below the threshold boron concentration (4 ppm).

Considering the values provided in Table 7, it was observed that current soils were appropriate for almost all crops including boron-sensitive cereals. Since boron toxicity does not exist in the region, there were no treats of boron for majority of crops cultivated within the region.

EC - Depth relationships based on the values provided in Table 7 are presented in Figure 9 and ESP Depth relationships are presented in Figure 10.EC values of soil layers did not change much, and values generally varied between 600-800 $\mu \mathrm{mhos} / \mathrm{cm}$ (Figure 9). Considering the water EC values of greater than limit value, it can be stated that salt accumulation in soils has not started, yet or salt leaching was well. However, such a case may generate a problem in the future.

It possible to state that ESP values also did not change much with the depths (Figure 10). ESP values of experimental soils were all below the threshold ESP value $(15 \%)$.

Table 6

Soil physical characteristics

\begin{tabular}{|c|c|c|c|c|c|c|c|c|c|}
\hline \multicolumn{2}{|c|}{ Soil Sample } & \multirow{2}{*}{$\begin{array}{c}\text { Degree of } \\
\text { Saturation } \\
(\%)\end{array}$} & \multirow{2}{*}{ Field Capacity (\%) } & \multirow{2}{*}{$\begin{array}{c}\text { Permanent } \\
\text { Wilting Point }(\%)\end{array}$} & \multirow{2}{*}{$\begin{array}{l}\text { Unit Weight } \\
\left(\mathrm{g} / \mathrm{cm}^{3}\right)\end{array}$} & \multicolumn{4}{|c|}{ Soil Texture } \\
\hline Plot No & Depth & & & & & Sand $\%$ & Clay \% & Silt \% & Texture \\
\hline \multirow{3}{*}{1} & $0-30$ & 51,2 & 33,9 & 18,7 & 1,42 & 26,3 & 46,9 & 26,8 & $\mathrm{C}$ \\
\hline & $30-60$ & 52,0 & 38,7 & 20,3 & 1,46 & 36,4 & 35,7 & 27,9 & CL \\
\hline & $60-90$ & 47,6 & 37,8 & 20,5 & 1,41 & 38,2 & 45,9 & 15,9 & $\mathrm{C}$ \\
\hline \multirow{3}{*}{2} & $0-30$ & 57,4 & 28,7 & 19,2 & 1,42 & 29,2 & 45,7 & 25,1 & $\mathrm{C}$ \\
\hline & $30-60$ & 46,2 & 33,6 & 21,3 & 1,39 & 38,6 & 36,5 & 24,9 & $\mathrm{CL}$ \\
\hline & $60-90$ & 47,4 & 36,9 & 20,6 & 1,37 & 25,5 & 53,4 & 21,1 & $\mathrm{C}$ \\
\hline \multirow{3}{*}{3} & $0-30$ & 46,4 & 26,1 & 14,2 & 1,45 & 30,7 & 48,0 & 21,3 & $\mathrm{C}$ \\
\hline & $30-60$ & 48,8 & 27,9 & 14,9 & 1,44 & 37,6 & 45,0 & 17,4 & $\mathrm{C}$ \\
\hline & $60-90$ & 49,6 & 28,7 & 15,3 & 1,41 & 42,8 & 45,5 & 11,7 & $\mathrm{C}$ \\
\hline \multirow{3}{*}{4} & $0-30$ & 52,4 & 30,2 & 17,6 & 1,49 & 26,3 & 49,8 & 23,9 & $\mathrm{C}$ \\
\hline & $30-60$ & 49,6 & 30,8 & 17,2 & 1,48 & 34,9 & 46,0 & 19,1 & $\mathrm{C}$ \\
\hline & $60-90$ & 53,0 & 31,1 & 18,4 & 1,45 & 26,9 & 51,3 & 21,8 & $\mathrm{C}$ \\
\hline \multirow{3}{*}{5} & $0-30$ & 57,2 & 31,2 & 19,7 & 1,37 & 42,5 & 34,7 & 22,8 & $\mathrm{CL}$ \\
\hline & $30-60$ & 56,8 & 35,6 & 19,4 & 1,39 & 38,7 & 36,4 & 24,9 & $\mathrm{CL}$ \\
\hline & $60-90$ & 54,8 & 37,9 & 20,2 & 1,38 & 38,8 & 38,1 & 23,1 & $\mathrm{CL}$ \\
\hline \multirow{3}{*}{6} & $0-30$ & 47,4 & 26,4 & 16,8 & 1,41 & 31,9 & 44,4 & 23,7 & $\mathrm{C}$ \\
\hline & $30-60$ & 47,4 & 27,9 & 15,9 & 1,36 & 36,9 & 34,4 & 28,7 & $\mathrm{CL}$ \\
\hline & $60-90$ & 49,8 & 31,1 & 18,7 & 1,39 & 32,8 & 49,8 & 17,4 & $\mathrm{C}$ \\
\hline
\end{tabular}

Table 7

Soil chemical characteristics

\begin{tabular}{|c|c|c|c|c|c|c|c|c|c|c|c|c|c|c|c|c|c|c|c|c|}
\hline \multicolumn{2}{|c|}{ Soil Sample } & \multirow{2}{*}{$\mathrm{pH}$} & \multirow{2}{*}{$\begin{array}{c}\mathrm{EC} \times 10^{6} \\
\mu \mathrm{mhos} / \mathrm{cm} \\
25^{\circ} \mathrm{C}\end{array}$} & \multicolumn{10}{|c|}{ Water Soluble } & \multirow{2}{*}{$\underset{(\mathrm{me} / 100 \mathrm{~g})}{\mathrm{CEC}}$} & \multicolumn{3}{|c|}{ Exchangeable Cations } & \multirow{2}{*}{$\begin{array}{l}\text { ESP } \\
(\%)\end{array}$} & \multirow{2}{*}{$\underset{(\%)}{\operatorname{Lime}}$} & \multirow{2}{*}{$\begin{array}{l}\text { Boron } \\
\text { (ppm) }\end{array}$} \\
\hline $\begin{array}{l}\text { Plot } \\
\text { No }\end{array}$ & Depth & & & $\mathrm{Na}^{+}$ & $\mathbf{K}^{+}$ & $\mathrm{Ca}^{+2}$ & $\mathrm{Mg}^{+2}$ & $\Sigma$ & $\mathrm{CO}_{2}^{-}$ & $\mathrm{HCO}_{3}$ & $\mathrm{Cr}$ & $\mathrm{SO}_{4}^{-2}$ & $\Sigma$ & & $\mathrm{Na}^{+}$ & $\mathbf{K}^{+}$ & $\mathrm{Ca}^{+2}+\mathrm{Mg}^{+2}$ & & & \\
\hline \multirow{3}{*}{1} & $0-30$ & 7,11 & 734 & 2,57 & 0,61 & 3,83 & 0,93 & 7,94 & - & 0,89 & 2,2 & 3,95 & 7,04 & 16,52 & 0,94 & 4,76 & 9,21 & 7,18 & 2,22 & 0,20 \\
\hline & $30-60$ & 7,25 & 734 & 2,57 & 0,81 & 3,21 & 0,47 & 7,06 & - & 0,97 & 2,1 & 3,85 & 6,92 & 16,21 & 1,03 & 3,62 & 12,54 & 7,05 & 1,89 & 0,80 \\
\hline & $60-90$ & 7,22 & 750 & 2,67 & 0,72 & 3,11 & 0,96 & 7,46 & - & 0,79 & 1,9 & 4,45 & 7,14 & 16,01 & 1,43 & 4,20 & 10,07 & 6,96 & 2,21 & 0,85 \\
\hline \multirow{3}{*}{2} & $0-30$ & 7,45 & 696 & 2,67 & 0,21 & 3,21 & 0,45 & 6,54 & - & 1,20 & 1,8 & 3,69 & 6,69 & 15,40 & 1,99 & 5,51 & 8,40 & 6,69 & 8,52 & 0,12 \\
\hline & $30-60$ & 7,57 & 727 & 2,57 & 0,82 & 3,20 & 0,55 & 7,14 & - & 1,40 & 2,0 & 3,76 & 7,16 & 15,50 & 1,38 & 4,13 & 10,65 & 6,74 & 9,62 & 0,49 \\
\hline & $60-90$ & 7,65 & 731 & 2,97 & 0,73 & 2,99 & 0,85 & 7,54 & - & 0,88 & 1,5 & 4,80 & 7,18 & 15,40 & 1,01 & 3,76 & 10,21 & 6,69 & 11,99 & 0,33 \\
\hline \multirow{3}{*}{3} & $0-30$ & 7,81 & 771 & 2,10 & 0,83 & 4,21 & 0,24 & 7,38 & - & 1,20 & 3,7 & 2,84 & 7,74 & 17,23 & 0,43 & 3,70 & 9,82 & 7,49 & 4,10 & 0,26 \\
\hline & $30-60$ & 7,90 & 736 & 2,52 & 0,95 & 3,21 & 0,65 & 7,33 & - & 0,99 & 3,2 & 2,96 & 7,15 & 15,50 & 0,75 & 3,63 & 11,99 & 6,74 & 10,57 & 0,23 \\
\hline & $60-90$ & 7,80 & 731 & 2,43 & 0,73 & 3,82 & 0,53 & 7,51 & - & 0,77 & 1,7 & 5,03 & 7,50 & 15,19 & 1,94 & 3,54 & 9,25 & 6,60 & 12,94 & 0,41 \\
\hline \multirow{3}{*}{4} & $0-30$ & 7,56 & 765 & 3,52 & 0,23 & 3,30 & 0,71 & 7,76 & - & 1,49 & 1,2 & 4,95 & 7,64 & 16,32 & 1,92 & 3,66 & 8,35 & 7,09 & 2,37 & 0,08 \\
\hline & $30-60$ & 7,60 & 762 & 3,60 & 0,12 & 3,53 & 0,21 & 7,46 & - & 1,60 & 1,0 & 4,69 & 7,29 & 16,72 & 1,62 & 3,23 & 11,89 & 7,27 & 1,58 & 0,14 \\
\hline & $60-90$ & 7,63 & 803 & 3,80 & 0,61 & 3,50 & 0,40 & 8,31 & - & 1,66 & 1,8 & 4,57 & 8,03 & 16,93 & 0,72 & 3,58 & 9,09 & 7,36 & 1,74 & 0,16 \\
\hline \multirow{3}{*}{5} & $0-30$ & 7,36 & 753 & 3,43 & 0,21 & 3,67 & 0,27 & 7,58 & - & 1,22 & 2,1 & 4,34 & 7,66 & 17,13 & 0,70 & 4,42 & 9,67 & 7,45 & 2,21 & 0,32 \\
\hline & $30-60$ & 7,44 & 775 & 3,53 & 0,51 & 3,41 & 0,47 & 7,92 & - & 0,69 & 2,3 & 4,14 & 7,13 & 16,32 & 1,20 & 4,65 & 10,29 & 7,09 & 2,21 & 0,17 \\
\hline & $60-90$ & 7,50 & 718 & 3,48 & 0,41 & 3,29 & 0,56 & 7,74 & - & 1,00 & 2,0 & 4,25 & 7,15 & 16,83 & 2,31 & 4,36 & 8,40 & 7,31 & 3,00 & 0,24 \\
\hline \multirow{2}{*}{6} & $0-30$ & 7,60 & 730 & 1,56 & 0,21 & 4,57 & 0,89 & 7,23 & - & 1,21 & 2,9 & 3,16 & 7,27 & 17,13 & 1,99 & 5,42 & 6,92 & 7,45 & 5,68 & 0,19 \\
\hline & $30-60$ & 7,61 & 752 & 1,84 & 0,70 & 4,40 & 0,89 & 7,83 & - & 1,17 & 1,9 & 4,34 & 7,41 & 16,72 & 1,81 & 3,42 & 9,42 & 7,27 & 5,99 & 0,32 \\
\hline
\end{tabular}




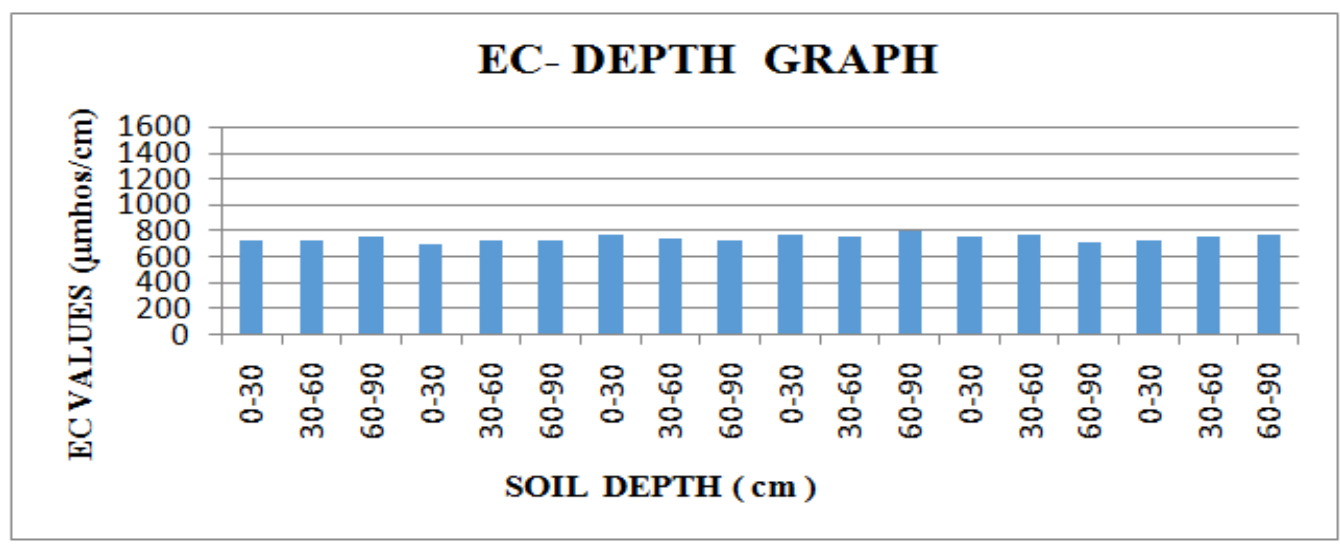

Figure 9

Soil EC - Depth relationships

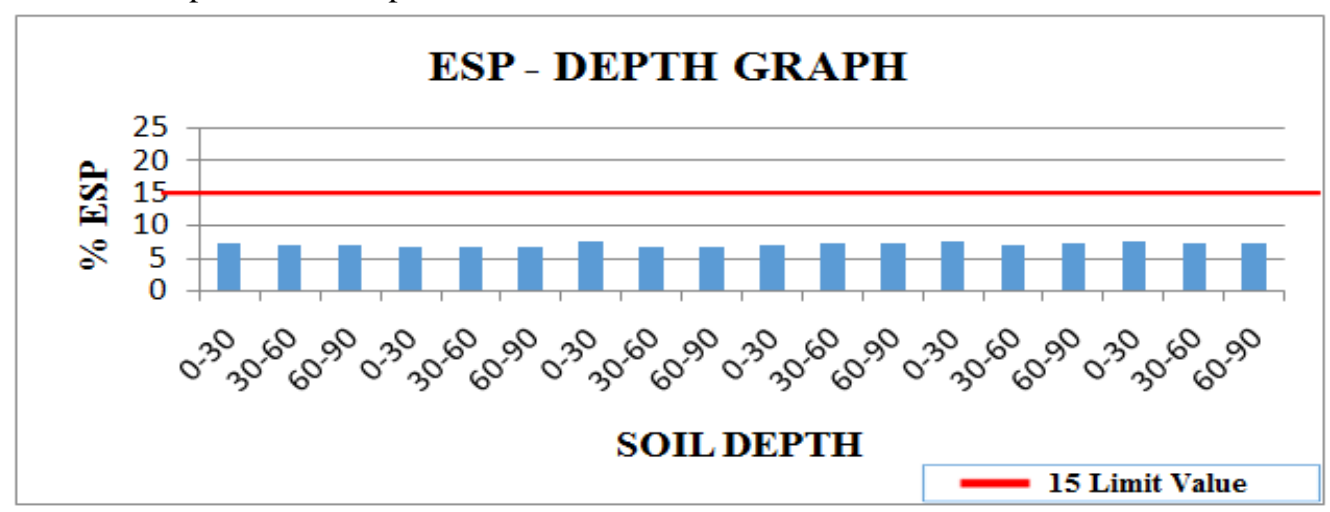

Figure 10

Soil ESP - depth relationships

Following conclusions were drawn based on present findings;

1) Considering irrigation water quality values in May, June, July, August and September all together, it was observed that water $\mathrm{pH}$ values varied between 6.08 - 7.45 and EC values varied between 1071-1989 $\mu \mathrm{mhos} / \mathrm{cm}$. Considering the EC values of the months, it was observed that EC values relatively increased from May to September and such increases were attributed to increasing plant water consumptions in summer season, consequent excessive water use and resultant decrease in well water levels and continuous use of saline waters. However, such increases did not change irrigation water class. With regard to water soluble cations and anions, it was observed that $\mathrm{Ca}^{+2}$ was the dominant cation and $\mathrm{HCO}_{3}{ }^{-}$and $\mathrm{SO}_{4}{ }^{-2}$ were the dominant anions. Sodium Adsorption Ratios (SAR) varied between $1.00-1.40, \% \mathrm{Na}$ values varied between 15.1 $-25.0 \%$ and boron concentrations varied between 0.15 $-0.36 \mathrm{ppm}$. Based on these values with regard to salinity and alkalinity, irrigation waters were classified as $\mathrm{C}_{3} \mathrm{~S}_{1}$ according to US Salinity Lab classification system. These waters could be used in pervious soils without any drainage problems. However, these waters should be avoided in clay soils with drainage problems or measures should be taken in case of use of these waters.

2) Boron concentrations all water samples were below the threshold boron concentration of $0.7 \mathrm{ppm}$.
Such a case also reflected in soil samples and boron toxicity (<4ppm) was not observed in soils.

3) Despite high salinity of well waters within the research site, salinity was observed in irrigated lands of the research site. Such a case was attributed to wellleaching or recent opening of the fields to irrigation. But, measures should be taken to prevent possible salinity problems in the future.

4) All of the water samples had greater salinity values than the threshold salinity level, thus it is possible to state that these waters should not be used in irrigations.

5) Soil degree of saturation values varied between 46.2 - 57.4\% and unit weights varied between $1.36-1.49 \mathrm{~g} / \mathrm{cm}^{3}$. Soils were mostly clay and clayloam in texture.

6) Soil $\mathrm{pH}$ values varied between 7.11 - 7.90, EC values varied between 696-803 $\mu \mathrm{mhos} / \mathrm{cm}$, cation exchange capacity (CEC) values varied between 15.19 17.23 me/100gr, exchangeable sodium percentage (ESP) values varied between $6.60-7.49 \%$, lime contents varied between $1.58-12.94 \%$ and boron concentrations varied between $0.08-0.85 \mathrm{ppm}$.

7) Exchangeable sodium percentage of all samples taken from the research site was below the threshold ESP value of $15 \%$.

8) Significant salt and boron accumulations haven't been reached, yet since sufficient time has not 
been elapsed for salt accumulation in soils by irrigations. Agricultural fields of the research site have been irrigated with groundwater resources for about 10 years. Common use of sprinkler irrigation might have prevented salt accumulation in soils.

\section{Recommendations}

1) According to present findings, irrigation water resources of the research site had salinity problems, however, salinity problem was not encountered in fields irrigated with these waters. That does not mean that salinity problem will not be encountered in near future since irrigation history of these fields are quite new. Quality of water resources of the region should be improved, and quality water should be delivered to fields as soon as possible.

2) Despite inexistence of salinity - alkalinity problems and considering the salinity problem of available water resources, it is recommended that drainage facilities should be constructed and land leveling should be performed in required places to prevent future salinity problems. Cultivation of salt-tolerant crops (barley, sugar beet) may also delay the emergence of a salinity problem.

3) Besides agricultural practices, reclamation practices should also be implemented over the agricultural fields of the region. Soil organic matter contents should be improved, and proper cropping patterns should be practiced.

4) Since the water resources of the region are replenished from the same reservoirs, farmers around the research site should be trained about water quality and salinity problems, significance and use of appropriate irrigation methods by Gözlü Agricultural Enterprise, agricultural organizations and universities.

5) While performing irrigations with saline waters, a certain leaching water fraction should be added to irrigationwater under appropriate drainage conditions. Local farmers should be informed about this issue.

6) There is a high possibility of soil salinity in near future since saline irrigation waters are still being used in irrigations. Therefore, either quality of water resources should be improved, or appropriate drainage facilities should be constructed.

\section{References}

Anliatamer E (2007). Surveying of the Irrigation Area of Ankara Haymana Türkşerefli Reservoir As Irrigation Parameters And Salinity, Ankara University Graduate School of Natural and Applied Sciences Department of Agricultural Structures and Irrigation Master Thesis.

Anonymous (2013). https://biruni.tuik.gov.tr/ bitkiselapp/bitkisel.zul (Access Date:21.06.2017) (In Turkish).
Anonymous (2016). http://www.tuik. gov.tr/il Gostergeleri/iller/KONYA.pdf (Access Date: 26.09.2017) (In Turkish).

Ayyıldız M (1983). Sulama Suyu Kalitesi ve Problemleri, Ankara Üniversitesi Ziraat Fakültesi Yayınlarl, No:876, Ders Kitabı 224, Ankara (In Turkish).

Dorak S and Çelik H (2017). Irrigation water quality of Nilüfer Stream and effects of the wastewater discharges of the treatment plants, Journal of Agricultural Faculty of Ege University, 2017, 54 (3):249257.

Gürcan S (2016). Evaluation of Water Resources in Terms of Irrigation Quality in Irrigated Areas of Ankara-Haymana-Soğulca Town Irrigation Cooperative, The Graduate School of Natural and Applied Science of Selçuk University Department of Agricultural Structures and Irrigation Master Thesis, Konya.

Husain S, Munns R, Condon AG (2003). Effect of Sodium Exclusion Trait on Chlorophyll Retention and Growth of Durum Wheat in Saline Soil, Australian Journal of Agricultural Research, 54 (589597).

Kara M (2005). Sulama ve Sulama Tesisleri, Selçuk Üniversitesi Ziraat Fakültesi Tarımsal Yapılar ve Sulama Bölümü, Konya (In Turkish).

Korkmaz N, Gündüz M, Aşık Ş (2016). Temporal variations in the levels and quality of groundwater in the agricultural areas in the South-East of the Gediz River, Derim, 33 (2):263-278.

Minareci Ö, Öztürk M (2012). Manisa İli Baraj Göllerinde Bor Kirliliğinin Araştırılması, Biyoloji Bilimleri Araştırma Dergisi, 5(1), s. 25-29, Manisa (In Turkish).

Sağlam T (1978). Toprak Kimyas1, Atatürk Üniversitesi Ziraat Fakültesi Uygulama Teksiri, Erzurum (In Turkish)

Taş İ, Yıldırım YE, Özkay F, Aras İ (2013). Konya Ovasında Su Kalitesi ve Toprak Tuzluluğu, Ulusal Kop Bölgesel Kalkınma Sempozyumu, 14-16 Kasım 2013, Konya (In Turkish)

Yeter T, Yurtseven E (2015). The Effects of Irrigation Water Quality and Leaching Requirements on Germination and Vegetative Growth of Alfalfa, Soil Water Journal, 4(1):36-42.

Yurtseven E (2016). Sulama Suyu Kalitesi ve Tuzluluk, Ankara Üniversitesi Ziraat Fakültesi Tarımsal Yapılar ve Sulama Bölümü, Açık Ders Malzemeleri (In Turkish). 